\title{
BMJ Open Comparison of the costs of HPV testing through community health campaigns versus home-based testing in rural Western Kenya: a microcosting study
}

To cite: Olwanda EE, Kahn JG, Choi Y, et al. Comparison of the costs of HPV testing through community health campaigns versus home-based testing in rural Western Kenya: a microcosting study. BMJ Open 2020;10:e033979. doi:10.1136/ bmjopen-2019-033979

- Prepublication history for this paper is available online. To view these files, please visit the journal online (http://dx.doi org/10.1136/bmjopen-2019033979).

Received 10 September 2019 Revised 29 July 2020 Accepted 21 September 2020

Check for updates

(C) Author(s) (or their employer(s)) 2020. Re-use permitted under CC BY-NC. No commercial re-use. See rights and permissions. Published by BMJ.

For numbered affiliations see end of article.

Correspondence to Easter Elizabeth Olwanda; eolwanda@gmail.com

\section{ABSTRACT}

Objectives To estimate the cost of human papillomavirus (HPV)-based screening through community health campaigns (CHCs) and home-based testing.

Setting $\mathrm{CHCs}$ and home-based testing in six communities in rural Western Kenya.

Participants CHCs and home-based screening reached 2297 and 1002 women aged 25-65 years, respectively. Outcome measures Outcome measures were overall cost per woman screened achieved through the CHCs and home-based testing and the cost per woman for each activity comprising the screening intervention.

Results The mean cost per woman screened through CHCs and home-based testing were similar, at \$37.7 (range \$26.4-\$52.0) and \$37.1 (range \$27.6-\$54.0), respectively. For CHCs, personnel represented $49 \%$ of overall cost, supplies $25 \%$, services $5 \%$ and capital goods $23 \%$. For home-based testing, these were: personnel $73 \%$, supplies $25 \%$, services $1 \%$ and capital goods $2 \%$. A greater number of participants was associated with a lower cost per participant.

Conclusions The mean cost per woman screened is comparable for $\mathrm{CHC}$ and home-based testing, with differences in type of input. The CHCs generally reached more eligible women in the six communities, whereas home-based strategies more efficiently reached populations with low screening rates.

Trial registration number NCT02124252.

\section{INTRODUCTION}

Cervical cancer is the fourth most common cancer among women, with an estimated 570000 new cases worldwide in 2018. ${ }^{1}$ The global age-standardised incidence rate is 14.1 per 100000 women-years, and three times higher in Kenya at $40.1^{2}$. Cervical cancer is almost entirely preventable through organised screening services, treatment of precancerous cervical lesions and vaccination against high-risk (oncogenic) human papillomavirus (HPV). ${ }^{3}$ The high incidence of cervical cancer in Kenya can be attributed largely to poor access to screening and low uptake of preventive services. ${ }^{4}$ Screening coverage is $3.2 \%$ for
Strengths and limitations of this study

- This study used prospective microcosting to estimate costs of two human papillomavirus screening strategies.

- This study estimated personnel time using time and motion method.

- The costing perspective is the health system, not considering costs to individuals.

- The analysis does not provide cost estimates for linkage and treatment.

- The analysis does not assess repeated implementation of these strategies

all women (4.0\% urban and $2.6 \%$ rural).$^{5}$ Although HPV vaccination programmes are scaling up globally, implementation has been slow and vaccination is not yet widely available in low-income and middle-income countries (LMICs), including Kenya. ${ }^{6}$ Further vaccination in most LMICs exclusively targets adolescent girls, leaving screening programmes the key cervical cancer prevention strategy for reproductive-aged women.

At the time we started, there were no screening services available in Migori, except episodic screening with Visual Inspection with Acetic Acid (VIA) done as part of limited outreach campaigns. In our primary paper, around $11 \%$ of women had reported ever having screening. ${ }^{7}$ The WHO recommends the use of HPV testing for primary cervical cancer screening, ${ }^{8}$ specifically in LMICs where the implementation of successful cytology-based screening programmes is not feasible due to costs and lack of healthcare infrastructure and trained pathologists. Self-collection of cervicovaginal samples for HPV testing has been shown to offer an inexpensive and effective way of improving screening coverage. ${ }^{9}$ However, the lack of finances for transport and long distances to 
health facilities are barriers to cervical cancer screening, including HPV self-collection. ${ }^{10} 11$

Our previous work shows that using community health campaigns (CHCs) to offer HPV testing through selfcollection can effectively reach underserved populations. ${ }^{12}$ Our recent cluster-randomised trial achieved a screening rate of $60 \%$ of the eligible population in rural Western Kenya, ${ }^{7}$ compared with a historical baseline of around $2.6 \% .^{5}$ An important advantage of self-collected HPV testing is that it removes barriers faced by women when offered clinic-based services. Home-based testing is an implementation strategy that has been shown to improve cervical cancer screening coverage. ${ }^{13}$ A population-based cluster-randomised trial in Argentina in which community health workers (CHWs) provided women with HPV selfcollection test kits at home resulted in a fourfold increase in screening uptake, with $86 \%$ uptake in the intervention group and compared with $20 \%$ uptake in the control group. ${ }^{14}$ Self-collection at CHCs and home-based testing can augment each other within the same programme and consequently raise cervical cancer screening uptake.

To plan the scale-up of HPV-based screening through CHCs and home visits, it is essential to understand implementation costs. These costs will also be critical inputs in cost-effectiveness analyses. Although prior studies estimated the costs of HPV screening for cervical cancer in clinics, ${ }^{15-18}$ the present study is the first to estimate the cost of HPV screening through CHCs and home-based testing in a LMIC. ${ }^{19}$ We present estimates of the direct costs of this programme in rural Western Kenya.

\section{MATERIALS AND METHODS \\ Study design}

This microcosting study was part of a two-phase clusterrandomised trial in Nyanza, Kenya, to determine the uptake rates of implementation strategies for HPV selftesting. Between February and October 2018, six rural communities were offered HPV screening through CHCs. We defined a community as one or two sublocations within a defined administrative boundary. Each community had a total population size of between 4500 and 9500 and had either a level II, III or IV Ministry of Health facility. Though each community had between 10 and 12 villages, we only measured costs at the community level.

Women aged between 25 and 65 years who did not screen at the CHCs (46.4\% of the target population) were offered home-based screening in November 2018. The implementation strategy for both CHCs and home-based testing consisted, in different intensities, of outreach and mobilisation, screening and notification of results (see figure 1).

In all communities, we informed all the eligible women about CHC-based screening first through community outreach. We then conducted a second outreach for the home-based testing to reach the women who did not screen at the CHCs (see figure 1) We offered HPV testing through self-collection to women from both CHCs and home-based testing.

Outreach for the CHCs was conducted for 2 weeks before the screening services were available and involved door-to-door mobilisation and meetings with key stakeholders. Resources used were the study vehicle, fuel and personnel, including 2 research assistants, 10 community health volunteers (CHVs), 1 study coordinator, 1 study driver and 1 study administrator. Resources used were similar across the six communities, except for two (Olasi and Osingo) where the study vehicle broke down, requiring transport reimbursement for the research assistants.

For home-based testing, outreach and mobilisation took place concurrently with screening. At least $10 \mathrm{CHVs}$ from each community, accompanied by the research assistants, identified the homes of eligible women who did not screen at the CHCs and offered them the HPV selfcollection kits to be completed at home.

Activities dedicated to screening included registration, group education, informed consent and HPV selfcollection. A multidisciplinary team that included experts in cervical cancer prevention, healthcare providers with knowledge of community strategies and CHVs with experience delivering health education in Kenya conceptualised and designed an education module. The education module was delivered before screening for both the CHCs and homes to educate the women on anatomy, definitions of cervical cancer and HPV, how screening works, how to conduct self-HPV testing, result interpretation and the available treatments. A positive test result meant having a type of high-risk HPV that is linked to cervical cancer. We strongly emphasised early treatment to prevent that progression to cervical cancer in the future. Follow-up test was recommended in a year or 3 years for HIV-positive and HPV-negative women, respectively, to see if the infection had cleared or to check for signs of cervical cancer. ${ }^{20}$

The HPV screening campaigns lasted 10 days per community for the CHCs and 4 days per community for home-based testing. The CareHPV testing system used was not a point-of-care test; therefore, the collected specimens were transported daily from the CHCs and homes to the study laboratory at Migori County Hospital for processing. The tests were run in batches of 90 , with a turnover time of approximately 1-2 weeks for the women from both sites to know their results. ${ }^{21}$ Options for notification of results included: home visits, text messaging and phone calls. However, there were implementation differences between the two strategies during notification. At the CHCs, both HPV-positive and HPV-negative women who opted for home visits were notified by the research assistants over 10 days per community. The study vehicle was used for transport during notification in four of the six communities. For women screened at home, the CHVs conducted home visits for HPV negative women, while the assistant study coordinator conducted home visits for the HPV-positive women using the study motorbike. 


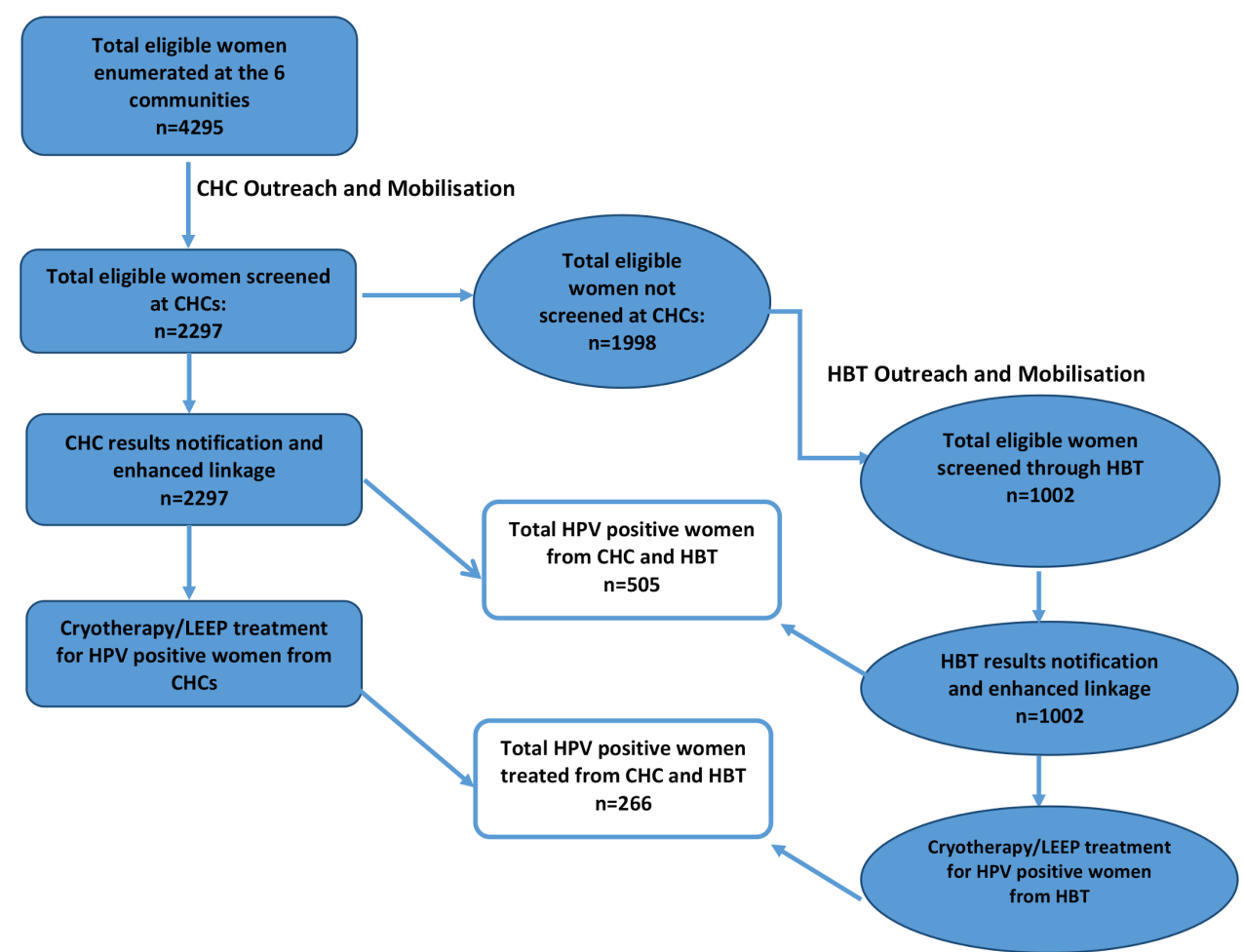

Figure 1 Diagram of the workflow for the CHCs and HBT. We offered HPV screening to eligible women from the six communities through $\mathrm{CHCs}$ first. $\mathrm{CHC}$ outreach and mobilisation was conducted through door-to-door mobilisation and meetings with key stakeholders. CHC-based screening then followed. Eligible women who did not screen at the CHCs were identified and offered home-based testing. The CHVs conducted door-to-door mobilisation followed by home-based screening. $\mathrm{CHC}$ participants received their results through frontline SMS, phone calls or home visits by research assistants. HPV-negative women from HBT were notified of their results at their homes by CHVs. The assistant study coordinator conducted home visits to deliver results to the HPV positive women. A total of 505 women from the CHC and HBT arms tested HPV positive. We then provided cryotherapy/LEEP treatment to $266 \mathrm{HPV}$-positive women from both $\mathrm{CHC}$ and HBT arms. CHCs, community health campaigns; HBT, home-based testing; HPV, human papillomavirus; LEEP, Loop Electrosurgical excision procedure.

The high-risk (hrHPV) positivity rate in this population was $17 \%$. A total of 505 women tested positive for hrHPV ${ }^{22}$ hrHPV-positive women from both the CHCs and home-based testing were referred to one of four government health facilities based on proximity to their community for a visual exam with acetic acid and treatment with cryotherapy/LEEP per the WHO guidelines. ${ }^{23}$ The government health facilities were Migori County Referral Hospital, Macalder Sub-County Hospital, Ogwedhi Health Centre and Karungu Sub-County Hospital. Timely and effective linkage to cryotherapy/LEEP treatment for both screening strategies was achieved by decentralisation of treatment centres, making follow-up phone calls and sending text message reminders to the hrHPV women who had received their HPV test result but had not yet accessed treatment within 1 month. These strategies were developed in collaboration with the Ministry of Health and based on feedback from healthcare providers and participants in the ongoing study. In addition, a study conducted to explore perceived health facility barriers to linkage and retention in an HIV care programme in Western Kenya confirmed that decentralisation of HIV care services right at the community level is critical to addressing poor linkage and retention rates. ${ }^{24} \mathrm{~A}$ study in Tanzania is also looking into whether SMS reminders will decrease loss to follow-up for treatment following a positive HPV screen. ${ }^{25}$

\section{Costing methods}

We applied microcosting methods from the provider's perspective to estimate the delivery cost of HPV screening in CHCs and home-based testing. We adopted an economic perspective, whereby all resources were costed at full value even if donated or subsidised. We enumerated the resources used, multiplied by the price paid or market quotes and summed to estimate the total cost in each community, and finally divided by the total number of screening participants to arrive at unit costs per woman screened. All costs are reported in US dollars, converted from Kenyan shillings at a commercial exchange rate of 101.7 Kenyan shillings per US dollar (17 January 2018). ${ }^{26}$

We extracted cost information from expenditure records and study logs, supplemented by interviews with administrative staff and the team that delivered the services at each site. For both the CHCs and home-based testing, we collected time and motion data daily on paperbased forms to estimate personnel time spent on CHC activities. ${ }^{27}$ We omitted time explicitly used for research, including regulatory activities and administering research questionnaires. 
We classified resources into four main input categories: personnel, recurrent supplies, services and capital goods. We estimated personnel compensation from project financial records. For staff with multiple responsibilities, we obtained information on the time dedicated to the interventions via interviews (eg, for outreach and notification activities), supplemented by time and motion data (collected during screening activities). When the two methods covered the same issue, for example, hours per week on different tasks, we relied on time and motion data, which was collected in real time. Recurrent supplies refer to items consumed within 1 year as well as longer lived resources of low value. These included careHPV (QIAGEN Inc, Gaithersburg, Maryland, USA) collection media, test kits and brushes, pipette tips, motor vehicle fuel and staff t-shirts. Services include expenditures on consultant fees, IT support, utilities and vehicle maintenance. We estimated the cost of recurrent supplies and services from expenditure records, and then conducted interviews with the staff to establish allocation across different functions and time periods. Capital goods and equipment are items with more than 1 year of useful life and value of $>\$ 250$; examples study vehicle, careHPV test system, study motorbike and tablets. Costs of capital goods were amortised on a $0 \%$ real discount rate basis over 5 years (useful life) assuming no salvage value.

Outcome measures were overall cost per woman screened achieved through the CHCs and homebased testing and the cost per woman for each activity comprising the screening intervention. The overall cost per woman screened was calculated by dividing the total cost of all six sites, designated for programme purposes, by population uptake of HPV-based screening.

\section{Patient and public involvement}

We did not involve patients in the identification or recruitment of participants. In addition, the patients did not assess the burden of the intervention. Our preliminary work was done in partnership with the Ministry of Health, which informed research questions and measures. The research assistants asked all participants to provide written informed consent to participate in the study before data collection. Consent for low-literacy participants was affirmed with a thumbprint. We communicated all individual screening results with all participants and disseminated the cost results through two key stakeholder meetings.

\section{RESULTS}

The mean cost per woman screened through the CHCs and home-based testing was $\$ 37.7$ (range \$26.4-\$52.0) and $\$ 37.1$ (range $\$ 27.6-\$ 54.0$ ), respectively (tables 1 and 2). For CHCs, personnel represented $48.7 \%$ of overall cost, supplies $24.7 \%$, services $5.5 \%$ and capital $23.2 \%$. For home-based testing, personnel represented $72.6 \%$ of the total cost, supplies $24.5 \%$, services $0.9 \%$ and capital goods $1.9 \%$.
Outreach and mobilisation activities cost $\$ 6.3$ per woman at the CHCs versus only $\$ 1.4$ for home-based testing due to implementation differences (figure 2). Home-based testing had at least 10 CHVs per community who were reimbursed at a daily rate of $\$ 4.9$ per CHV. The home-based testing strategy reported cost savings attributed to the lean personnel team required for outreach and the fact that fuel and study vehicle was not needed.

HPV screening campaigns cost $\$ 23.5$ and $\$ 19.3$ per woman at the CHCs and home-based testing, respectively. Personnel cost per woman was higher for the home-based testing (\$9.6) compared with the CHCs (\$9.3). Homebased testing employed four extra field assistants to meet the additional screening demand within a month's timeline. Recurrent supplies were $\$ 9.0$ per woman for the home-based testing and \$8.1 per woman for the CHCs due to increased fuel costs required to travel to each participant's homes to conduct the intervention. The capital cost per woman at the CHCs and the home-based testing was $\$ 4.3$ and $\$ 0.6$, respectively. Cost savings were observed for home-based testing because resources such as tents, tables and chairs were not required. Additionally, operations and maintenance services such as tent assembly, security, mobilisation and car hire during screening were not required for home-based testing. The mean service cost for the pick-up hired to transport tents, chairs and tables to the CHCs was $\$ 1.9$ per woman.

The notification cost per woman was higher for homebased testing (\$16.3) compared with CHCs (\$7.9). We observed a threefold rise in personnel costs for homebased testing notification since extra personnel effort was required to physically locate the HPV-positive women at their homes and make return visits for those not found. Capital cost was higher for the CHCs (\$2.4) compared with the home-based testing $(\$ 0.1)$. The study vehicle was used to conduct home visits for CHC participants, while the study motorbike was used to reach the participants of home-based testing. Consequently, the cost of recurrent supplies for notification of home-based participants also reduced due to the fuel requirements for the study motorbike (\$0.1 per woman notified) compared with that of the study vehicle (\$0.2 per woman notified).

We also observed a significant relationship ( $p$ value $=0.0009$ ) between overall cost per woman screened and higher numbers of women screened.

\section{DISCUSSION}

To our knowledge, this is the first study to directly compare the cost of HPV screening offered through community health campaigns to a model of home-based testing. The mean cost per woman screened from the CHCs (\$37.7) is comparable with that of home-based testing (\$37.1), though relying on a different mix of input resources and activities. These findings are important for cervical cancer screening programmes, exemplifying the need to explore 


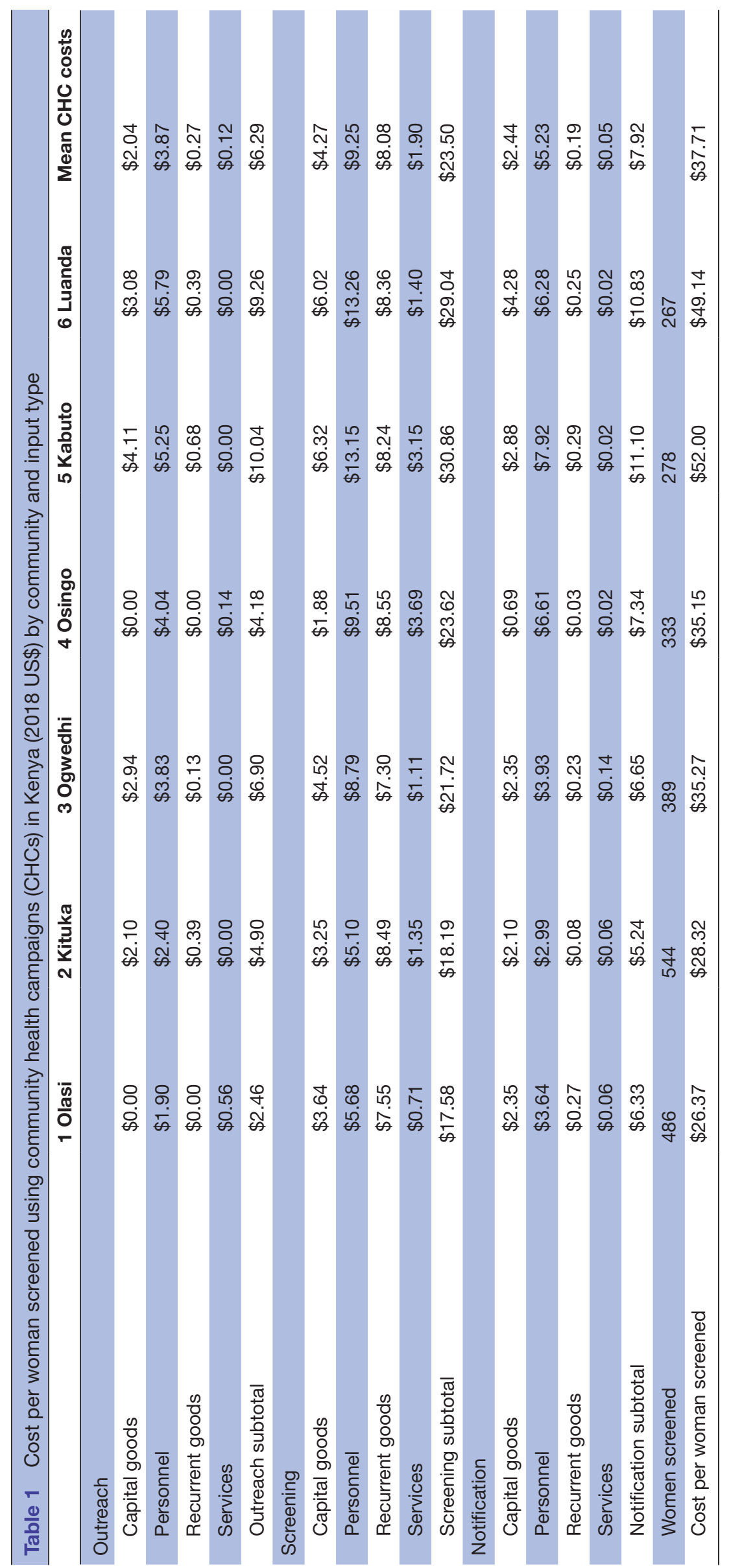

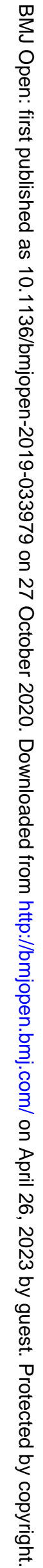


Table 2 Cost per woman screened using home-based testing in Kenya (2018 US\$) by community and input type

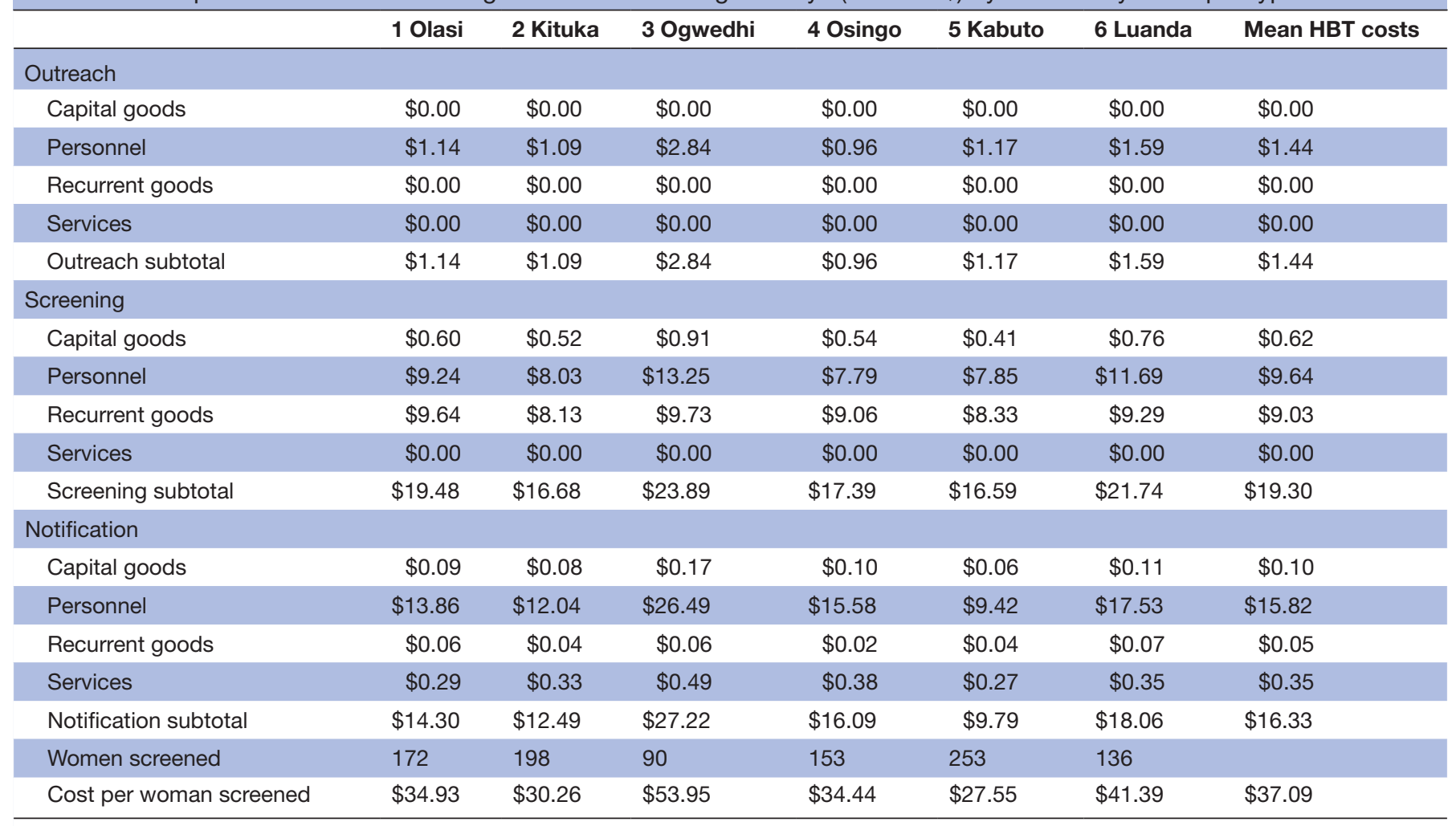

HBT, home-based testing.

and implement a variety of health delivery strategies to reach the largest number of women.

Decrease in cost per woman screened may be possible through economies of scale by spreading fixed costs

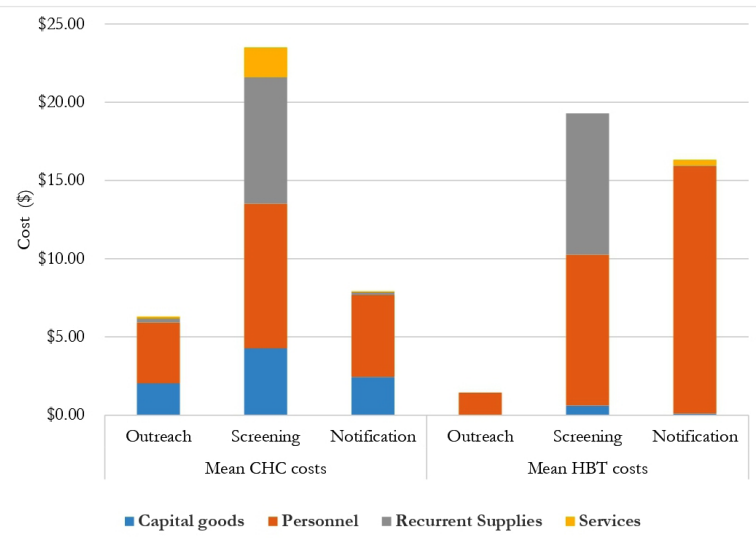

Figure 2 Cost per woman screened at $\mathrm{CHCs}$ and homebased testing colour-coded by activity and input type. The mean cost of outreach and mobilisation and screening was higher at the $\mathrm{CHCs}$ compared with the home-based testing. The mean cost per woman notified through the home-based testing was higher compared with the CHCs. The mean recurrent costs per woman screened were higher for the home-based testing compared with the CHCs. The mean capital cost per woman screened at the CHCs was higher than home-based testing. The mean service and personnel costs per woman notified higher for those who screened through home-based testing compared with the $\mathrm{CHCs}$. $\mathrm{CHCs}$, community health campaigns. (capital goods and equipment) over increased screening participants, potential lower prices through bulk purchases, sharing of services and reduced personnel downtime. Recent studies have found that large-scale HIV prevention and treatment programmes are associated with decreased unit costs when scaled up, across multiple countries. ${ }^{28}{ }^{29}$ Implementers should also be aware of potential diseconomies that may arise from overcrowded CHCs, longer wait times and disenrolment, and provider burnout due to expanding the screening coverage in a very resource-constrained setting. ${ }^{27}$ Policymakers may also ask about cost-effectiveness of community health campaigns and home-based testing in local contexts. Future studies to evaluate cost savings with these strategies and to translate the cost per person testing HPV positive in screening programmes into cost-effectiveness (ie, cost per disability-adjusted life year averted) are needed.

Some prior studies have reported more significant differences in mean cost per person screened through CHCs versus home-based strategies, due to differences in implementation. For example, evaluations of HIV screening among hard-to-reach populations in rural sub-Saharan Africa have found home-based testing to be substantially more expensive than CHC and facility-based testing, primarily when used for CHC non-attendees. ${ }^{30} 31$ However, our findings are consistent with other studies that found the cost of home-based HIV testing to be lower than facility-based testing. ${ }^{32-34}$ Several factors that reduce the costs of home-based strategies include the use of low 
cost equipment such as motorbikes instead of a motor vehicle for results notification and low demand for operations and maintenance services such as tent assembly, security, mobilisation and car hire during screening. A study in Uganda to evaluate the cost-effectiveness of facility-based and home-based voluntary HIV counselling and testing models reported substantial cost savings for the cost of operations and maintenance of buildings for home-based testing (\$0) compared with facility-based testing $(\$ 767){ }^{34}$ Low-cost strategies have the potential to make home-based HPV testing less expensive. When programme implementers consider context-specific screening needs, these findings will help them avoid design efficient delivery strategies.

Personnel costs were modest for both strategies, $\$ 9.6$ for home-based testing and $\$ 9.3$ for CHCs. Our findings are consistent with studies that found personnel costs for home-based testing of HIV programmes to be higher than that of $\mathrm{CHCs}^{30}$ and facilities. ${ }^{34}$ Both strategies achieved lower personnel costs, using different approaches. The CHCs reduced personnel downtime (and thus costs) through task shifting when the CHCs were crowded. Using CHVs reduced the personnel cost of implementing homebased testing significantly since their compensation was one-third that of the research assistants. Previous research has demonstrated that task shifting, including the use of CHWs to deliver care, can improve population health and is a viable option for health systems cost savings on LMICs. ${ }^{35}$ Our findings suggest that there is potential for HPV screening to integrate into CHVs' regular home visits instead of being offered by the screening-specific team at the CHCs. Personnel costs for notification of home-based participants were three times higher than that of the CHCs because of practical challenges, for example, with every effort made to retrace hard-to-reach participants. Our data suggest that notification of home-based testing participants can be planned systematically, streamlining processes and preventing unnecessary duplication of effort.

Our study has several limitations. First, it does not estimate costs for linkage and treatment. Although this underestimates the total cost of cervical cancer prevention, our estimates are designed to be of direct budgetary and programmatic relevance to sites HPV screening through CHCs and home-based testing. Second, personnel costs did not include the costs required to recruit, retain and deploy health workers to the areas where they are most needed. Thus, the cost projections are conservative. Finally, we evaluated a model of home-based testing after CHCs, not a standalone home-based testing model. While it is directly applicable to programmes looking to diversify HPV screening options and share screening resources, such as mobilisation, training and notification methods, the costs cannot be directly applied to a standalone campaign of home-based testing. There are scenarios in which an independent model would be more expensive (more personnel time and effort required to reach the eligible population; intense mobilisation) ${ }^{33}$ or less costly (through a higher number of women reached; a single type of training). ${ }^{36}$
Implementers and policymakers considering the expansion of cervical cancer screening in low-resource countries should take this into account for national programming. The analysis methods can be replicated in other programmatic and geographic settings.

\section{CONCLUSIONS}

Settings may benefit from programming options contextualised to meet the needs of their populations. We have shown that home-based and CHC-based testing have similar costs. Analogous programmes from the HIV field show potential strategies to reduce further the costs of home-based strategies, including motorcycles, personnel streamlining and integration into other home-based services. This should give programme planners more confidence to explore creative, responsive programmes to best meet the needs of their populations. Scale-up paired with effective low-cost community-based linkage to cryotherapy and LEEP treatment interventions are the essential next steps for these promising strategies.

\section{Author affiliations}

${ }^{1}$ Center for Microbiology Research, Kenya Medical Research Institute, Nairobi, Kenya

${ }^{2}$ Department of Epidemiology and Biostatistics, University of California, San Francisco, CA, USA

${ }^{3}$ Philip R. Lee Institute for Health Policy Studies, University of California, San Francisco, San Francisco, CA, USA

${ }^{4}$ Duke Global Health Institute, Duke University, Durham, NC, USA

${ }^{5}$ Duke University, Department of Obstetrics and Gynecology, Durham, NC, USA ${ }^{6}$ Department of Epidemiology, Gillings School of Global Public Health, University of North Carolina at Chapel Hill, Chapel Hill, NC, USA

${ }^{7}$ Lineberger Comprehensive Cancer Center, University of North Carolina at Chapel Hill, Chapel Hill, NC, USA

Twitter Easter Elizabeth Olwanda @easter_olwanda and Jessica Yasmine Islam @ jyislam

Acknowledgements We are grateful to the US National Institutes of Health/ National Cancer Institute for funding this study. We are thankful for the costing team at the Kenya Medical Research Institute, specifically Evans Otieno, who was the costing assistant, for his dedication and ensuring the success of the programme. We also acknowledge all investigators, patient advisers and community health volunteers who participated in the cervical cancer screening and prevention cluster randomised trial.

Contributors EEO designed the data collection procedures and collected the costing data, wrote drafts of the paper, verified analyses and reviewed the draft. JGK developed the costing model, advised on data collection design, data analyses, interpreted results and edited the manuscript. JYI advised on analysis, organisation and revision of the final manuscript. YC assisted with organisation and revision of the final manuscript. MH provided overall guidance in the costing study design, analysis and interpretation. All authors reviewed and approved the final version of the manuscript.

Funding This study was funded by the National Cancer Institute (R01-CA188428). Competing interests None declared.

Patient and public involvement Patients and/or the public were not involved in the design, or conduct, or reporting, or dissemination plans of this research.

Patient consent for publication Not required.

Ethics approval We have obtained IRB approval from the Kenya Medical Research Institute (KEMRI), Duke University, and University of California - San Francisco. The identification for KEMRI is SSERU 2918, for Duke is Pro0007742, and for UCSF is CHR 14-13698.

Provenance and peer review Not commissioned; externally peer reviewed. 
Data availability statement Data are available in a public, open access repository. Extra data can be accessed via the Dryad data repository at http://datadryad.org/ with the doi: 10.5061/dryad.tht76hdxf.

Open access This is an open access article distributed in accordance with the Creative Commons Attribution Non Commercial (CC BY-NC 4.0) license, which permits others to distribute, remix, adapt, build upon this work non-commercially, and license their derivative works on different terms, provided the original work is properly cited, appropriate credit is given, any changes made indicated, and the use is non-commercial. See: http://creativecommons.org/licenses/by-nc/4.0/.

\section{ORCID iDs}

Easter Elizabeth Olwanda http://orcid.org/0000-0002-3158-9415

Jessica Yasmine Islam http://orcid.org/0000-0002-3690-3848

\section{REFERENCES}

1 WHO. Cervical cancer. WHO, 2018. Available: https://www.who. int/cancer/prevention/diagnosis-screening/cervical-cancer/en/ [Accessed 15 Apr 2019].

2 ICO. Kenya: human papillomavirus and related cancers, fact sheet 2017 (ICO information centre on HPV and cancer)., 2017. Available: http://www.hpvcentre.net/statistics/reports/KEN_FS.pdf [Accessed 21 Mar 2018].

3 National Guidelines for Prevention. National guidelines for prevention and management of cervical, breast and prostate cancers. Available: https://www.k4health.org/sites/default/files/National Guidelines for Prevention and Management of Cervical\%2C Breast and Prostate Cancers.pdf [Accessed 21 Mar 2018].

4 Mbaka P, Waihenya R, Oisebe C. Factors affecting the uptake of cervical cancer screening in Mama Lucy Kibaki Hospital, Nairobi, Kenya. Cancer Res J 2018;6:106.

5 Bruni L, Barrionuevo-Rosas L, Albero G, et al. ICO information centre on HPV and cancer (HPV information centre). Hum papillomavirus Relat Dis world Summ Rep 2015;4.

6 Bruni L, Diaz M, Barrionuevo-Rosas L, et al. Global estimates of human papillomavirus vaccination coverage by region and income level: a pooled analysis. Lancet Glob Health 2016;4:e453-63.

7 Huchko MJ, Ibrahim S, Blat C, et al. Cervical cancer screening through human papillomavirus testing in community health campaigns versus health facilities in rural Western Kenya. Int $J$ Gynaecol Obstet 2018;141:63-9.

8 WHO. WHO Guidelines for Screening and Treatment of Precancerous Lesions for Cervical Cancer Prevention [Internet]. WHO Guidelines for Screening and Treatment of Precancerous Lesions for Cervical Cancer Prevention. World Health Organization, 2013.

9 Lorincz A, Castanon A, Wey Lim AW, et al. New strategies for human papillomavirus-based cervical screening. Womens Health 2013;9:443-52.

10 Ndejjo R, Mukama T, Kiguli J, et al. Knowledge, facilitators and barriers to cervical cancer screening among women in Uganda: a qualitative study. BMJ Open 2017;7:e016282.

11 Munthali AC, Ngwira BM, Taulo F. Exploring barriers to the delivery of cervical cancer screening and early treatment services in Malawi: some views from service providers. Patient Prefer Adherence 2015;9:501

12 Swanson M, Ibrahim S, Blat C, et al. Evaluating a community-based cervical cancer screening strategy in Western Kenya: a descriptive study. BMC Womens Health 2018;18:116.

13 Sultana F, Mullins R, English DR, et al. Women's experience with home-based self-sampling for human papillomavirus testing. BMC Cancer 2015;15:849.

14 Arrossi S, Thouyaret L, Herrero R, et al. Effect of self-collection of HPV DNA offered by community health workers at home visits on uptake of screening for cervical cancer (the EMA study): a population-based cluster-randomised trial. Lancet Glob Health 2015;3:e85-94.

15 Goldie SJ, Gaffikin L, Goldhaber-Fiebert JD, et al. Cost-Effectiveness of cervical-cancer screening in five developing countries. $N$ Engl J Med 2005;353:2158-68.
16 Campos NG, Mvundura M, Jeronimo J, et al. Cost-Effectiveness of HPV-based cervical cancer screening in the public health system in Nicaragua. BMJ Open 2017;7:e015048

17 Zimmermann MR, Vodicka E, Babigumira JB, et al. CostEffectiveness of cervical cancer screening and preventative cryotherapy at an HIV treatment clinic in Kenya. Cost Eff Resour Alloc 2017;15:13.

18 Sinanovic E, Moodley J, Barone MA, et al. The potential costeffectiveness of adding a human papillomavirus vaccine to the cervical cancer screening programme in South Africa. Vaccine 2009;27:6196-202

19 Shen J, Olwanda E, Kahn JG, et al. Cost of HPV screening at community health campaigns (CHCs) and health clinics in rural Kenya. BMC Health Serv Res 2018;18:378.

20 Choi Y, Oketch SY, Adewumi K, et al. A Qualitative Exploration of Women's Experiences with a Community Health Volunteer-Led Cervical Cancer Educational Module in Migori County, Kenya. $J$ Cancer Educ. 2018;35.

21 Huchko MJ, Kahn JG, Smith JS, et al. Study protocol for a clusterrandomized trial to compare human papillomavirus based cervical cancer screening in community-health campaigns versus health facilities in Western Kenya. BMC Cancer 2017;17:826.

22 Page CM, Ibrahim S, Park LP, et al. Patient factors affecting successful linkage to treatment in a cervical cancer prevention program in Kenya: a prospective cohort study. PLoS One 2019;14:e0222750.

23 Organization WH. Comprehensive cervical cancer prevention and control-a healthier future for girls and women: who guidance note. who Recomm use HPV test Prim screen also Recomm prolong screen interval to up to. 10. Geneva, 2013.

24 Wachira J, Naanyu V, Genberg B, et al. Health facility barriers to HIV linkage and retention in Western Kenya. BMC Health Serv Res 2014;14:646.

25 Linde DS, Andersen MS, Mwaiselage JD, et al. Text messages to increase attendance to follow-up cervical cancer screening appointments among HPV-positive Tanzanian women

(Connected2Care): study protocol for a randomised controlled trial. Trials 2017;18:555.

26 CBK. CBK exchange rates. Available: https://www.centralbank.go. ke/forex/ [Accessed 6 Dec 2018].

27 Olwanda E, Shen J, Kahn JG, et al. Comparison of patient flow and provider efficiency of two delivery strategies for HPV-based cervical cancer screening in Western Kenya: a time and motion study. Glob Health Action 2018;11:1451455.

28 Marseille E, Dandona L, Marshall N, et al. Hiv prevention costs and program scale: data from the PANCEA project in five low and middleincome countries. BMC Health Serv Res 2007;7:108.

29 Galárraga O, Wamai RG, Sosa-Rubí SG, et al. HIV prevention costs and their predictors: evidence from the ORPHEA project in Kenya. Health Policy Plan 2017;32:1407-16.

30 Chang W, Chamie G, Mwai D, et al. Implementation and operational research: cost and efficiency of a hybrid mobile Multidisease testing approach with high HIV testing coverage in East Africa. J Acquir Immune Defic Syndr 2016;73:e39-45.

31 Labhardt ND, Motlomelo M, Cerutti B, et al. Home-Based versus mobile clinic HIV testing and counseling in rural Lesotho: a clusterrandomized trial. PLoS Med 2014;11:e1001768.

32 Tabana $\mathrm{H}$, Nkonki L, Hongoro $\mathrm{C}$, et al. A cost-effectiveness analysis of a home-based HIV counselling and testing intervention versus the standard (facility based) HIV testing strategy in rural South Africa. PLoS One 2015;10:e0135048.

33 Menzies N, Abang B, Wanyenze R, et al. The costs and effectiveness of four HIV counseling and testing strategies in Uganda. AIDS 2009;23:395-401.

34 Mulogo EM, Batwala V, Nuwaha F, et al. Cost effectiveness of facility and home based HIV voluntary counseling and testing strategies in rural Uganda. Afr Health Sci 2013;13:423-9.

35 Seidman G, Atun R. Does task shifting yield cost savings and improve efficiency for health systems? A systematic review of evidence from low-income and middle-income countries. Hum Resour Health 2017;15:29.

36 Terris-Prestholt F, Kumaranayake L, Ginwalla R, et al. Integrating tuberculosis and HIV services for people living with HIV: costs of the Zambian protest initiative. Cost Eff Resour Alloc 2008;6:2. 